\title{
Une analyse sémio-poétique : «Le ciel est, par-dessus le toit » de Verlaine
}

\section{A Semio-poetic Analysis: « Le ciel est, par-dessus le toit » by Verlaine}

\author{
Ece KORKUT ${ }^{1}[\mathbb{C}$
}

'Prof. Dr., Hacettepe University, Faculty of Education, French Language Education Department, Ankara, Turkey

\section{ORCID: E.K. 0000-0002-1670-8957}

\section{Corresponding author:}

Ece KORKUT,

Hacettepe University, Faculty of Education, French Language Education Department, Ankara, Turkey

E-mail: ekorkut@hacettepe.edu.tr

Submitted: 26.03 .2020

Revision Requested: 04.04.2020

Last Revision Received: 17.04 .2020

Accepted: 22.04 .2020

Citation: Korkut, E. (2020). Une analyse sémio-poétique : « Le ciel est, par-dessus le toit » de Verlaine. Litera, 30(1), 27-43 https://doi.org/10.26650/LITERA2020-0059

\begin{abstract}
RÉSUMÉ
Le langage poétique se distingue d'autres types d'expression par sa syntaxe discontinue et elliptique ainsi que par son contenu inhabituel et métaphorique. Tandis que le langage pratique a une fonction essentiellement informative et communicative, le souci du langage poétique n'est pas prioritairement la communication. Quoique ce langage créatif soit utilisé dans d'autres domaines comme la publicité et la politique, son usage dans la poésie a une fonction dominante artistique, donc autonome. Par ailleurs un texte poétique s'éloigne de la prose à plusieurs égards. C'est la raison pour laquelle un poème ne peut être analysé comme on analyse d'autres types de textes. Cet article vise à analyser du point de vue formelle et sémantique un poème de Paul Verlaine, "Le ciel est, par-dessus le toit ». Pour montrer l'organisation rythmique du poème, l'analyse commence par le niveau de la forme avec les dispositions des rimes, le système métrique, l'allitération, l'assonance, l'homonymie, la paronymie. Cette analyse formelle débouche sur le niveau sémantique et notionnel avec des parasynonymes, les oppositions (spatiale, thymique, temporelle, nature/culture, les autres/je). Ce qui suit par l'analyse de tous les rapports référentiels entre les mots ou syntagmes de la poésie. Toujours au niveau du contenu, l'état et la place du /JE/ sont présentés en partant de ce qui précède. Ce poème composé de quatre quatrains est divisé en deux séquences : les trois strophes décrivent l'espace extérieur avec tous ses éléments euphoriques alors que dans la dernière strophe, c'est le /JE/ qui apparait dans son univers intérieur, coupé de l'espace ouvert, qui est entièrement dysphorique.
\end{abstract}

Mots-clés: Analyse poétique, euphorie, dysphorie, oppositions notionnelles, Verlaine

\section{ABSTRACT}

Poetic language is distinguished from other types of expression by its discontinuous and elliptical syntax as well as by its unusual and metaphorical content. While practical language has an essentially informative and communicative function, poetical language is not primarily concerned with communication. Although this creative language is used in other fields such as advertising and politics, its use in poetry has a dominant artistic function, therefore autonomous. Moreover, a poetic text deviates from prose in several respects. This is why a poem cannot be analyzed 
in the same way as other types of texts are analyzed. This article aims to analyze from a formal and semantic point of view a poem by Paul Verlaine, "Le ciel est, par-dessus le toit". To show the rhythmic organization of the poem, the analysis begins with the level of the form with the provisions of rhymes, the metric system, alliteration, assonance, homonymy, paronymy. This formal analysis leads to the semantic and notional level with parasynonyms, oppositions (spatial, thymic, temporal, nature / culture, others / I). This is followed by the analysis of all the referential relationships between the words or syntagms of poetry. Also, at the content level, the status and place of the /// are presented on the basis of the above. This poem composed of four stanzas is divided into two sequences: the three stanzas describe the outer space with all its euphoric elements while in the last stanza, the /I/ which appears in its inner universe, is cut off from the open space, which is entirely dysphoric.

Keywords: Poetic analysis, euphoria, dysphoria, notional oppositions, Verlaine

\section{EXTENDED ABSTRACT}

Unlike practical and ordinary language, the syntax of poetic language is discontinuous and elliptical. While the objective of practical language is to ensure communication, poetic language aims to create a certain effect on the recipient through an esthetic expression. Besides, poetry is opposed to prose which is linear, by its textual structure and by its rhythm. In other words, in poetry, the sound form takes precedence over the meaning. In this regard, Malherbe and Valéry equated prose with walking and poetry with dance.

This article aims to make a semio-poetic analysis of the poem by Paul Verlaine: «Le ciel est, par-dessus le toit « published in the collection Sagesse (1881). In terms of form, this fixed-form poem has remarkable regularity. In the arrangement of rhymes, the disposition of male and female rhymes as well as the richness of the rhymes, we observe a crossed form. As for the metric system, the crossed form is noted by verses of 8 syllables followed by verses of 4 syllables, from the beginning to the end of the poem. The reiterations of the signifier are carried out by a strong occurrence of several consonants (alliteration) and vowels (assonance). On the one hand, these phonemic reiterations assure the rhythm, on the other hand, they give clues to the content of the poem. Among the 57 words that make up the poem, there are also several homonyms and paronyms.

In terms of meaning, this poem can be divided into two sequences: the first 3 stanzas describe the outside world euphorized by all its constituent elements, while the last stanza is reserved for the inside world where the poetic subject is found. Still at the level of meaning, there are some reiterations of the signified ( 3 parasynonyms) and several repetitions of the same signs. What is particularly noteworthy is that this poem 
by Verlaine contains many semantic and notional oppositions. The first opposition emerges between the outdoor space and the indoor space. This spatial opposition is linked to the tyhmic opposition because the outer space presents itself as euphoric while the inner space is entirely dysphoric. The third opposition is temporal: the past is linked to the youth of the poetic subject ("qu'as-tu fait [...] de ta jeunesse?"), while the present time expresses the end of youth, and both have dysphoric value. Another notional opposition is established between nature and culture: the number of cultural elements in the poem is almost double that of natural elements. Between the others and the poetic subject, there is a final opposition. All these 5 oppositions, which are linked to each other, have been shown in tables and have been explained by elements taken from the text. In addition, the network of semantic and semiotic relationships between different signs of the text ensures the relevance carried out by associative fields. Thus, several signs and syntagms are linked by echoing each other. This relational network has been shown and explained by common semes: "berce - chante" (/rhythm/) ; "tinte - chante - rumeur" (/hearing sense/); "plainte - pleurant" (/dysphoria/); "cloche" - /église/ -"Dieu" (/religion/) ;"calme - berce - doucement - simple - tranquille - paisible" (/euphoria/) ; "la vie est là" - "toi que voilà" (/space/). Subsequently, the state and place of the poetic subject was clarified starting from the encompassing space (open, exterior, euphoric), the encompassed space (open, exterior, euphoric) and the enclosed space (interior, dysphoric) where the poetic subject is.

Style figures are frequent in the use of poetic language as well as in poems. Verlaine used in this little poem two metaphors and an oxymoron in verses 2, 3 and 4.

In conclusion, alongside the remarkable musicality in all of Verlaine's work, it is notably by the structure based on oppositions on the semantic and notional levels that we can characterize this poem. 


\section{Introduction}

Le langage poétique se distingue du langage ordinaire (et prosaïque) à plusieurs égards, tout d'abord par sa syntaxe discontinue et elliptique qui va à l'encontre de celle du langage linéaire. A ce propos, Malherbe ${ }^{1}$ assimilait la prose à la marche et la poésie à la danse en évoquant de la sorte que la poésie n'a d'autre objectif extérieur qu'ellemême. Paul Valéry qui a repris cette analogie dans « Propos sur la poésie » l'a explicitée de la manière suivante : «La marche comme la prose a toujours un objet précis. Elle est un acte dirigé vers quelque objet que notre but est de joindre. [...] La danse, c'est tout autre chose. Elle est, sans doute, un système d'actes, mais qui ont leur fin en euxmêmes. Elle ne va nulle part » $(1928$, p. 1371).

Cet usage particulier de la langue, qui s'écarte de la seule fonction communicative du langage (pratique) et qui la dépasse, se manifeste aussi bien dans la poésie qui est un domaine artistique que dans d'autres domaines comme la publicité et la politique, visant à créer un effet de sens. Néanmoins, comme l'affirme Jakobson, « l'œuvre poétique doit en réalité se définir comme un message verbal dans lequel la fonction esthétique est la dominante » (1973, p. 147).

Ce qui distingue la poésie d'autres domaines d'usage du langage poétique, c'est que la poésie se suffit à elle-même sans mettre en avant le souci communicationnel ou matériel. Bien que le point commun entre ces trois domaines (poésie, publicité, politique) soit de pouvoir créer un certain effet (perlocutoire) sur les destinataires -pour des raisons différentes-, contrairement au slogan publicitaire ou politique, la poésie n'a pour objectif que d'influencer les lecteurs ou auditeurs par sa structure sonore (formelle) plus que par son contenu (sémantique). C'est donc par le statut prioritaire du « signifiant » que s'éloigne la poésie de tous les autres types d'expression et de texte. Toute proportion gardée, à l'instar de la formule célèbre de Sartre, « l'existence précède l'essence » (Sartre, 1970, pp. 17-24), pour la poésie, on pourrait avancer que « la forme précède le sens » ou encore « la forme l'emporte sur le sens ». C'est dire que contrairement aux types de texte dits narratif, descriptif, explicatif, argumentatif, un texte poétique se caractérise avant tout par sa structure sonore, plus précisément par son organisation harmonieuse et rythmique. Pour Jakobson, « en poésie, toute similarité apparente dans le son est évaluée en termes de similarité et/ou dissimilarité dans le sens » (1963, p. 240). Il en découle que l'on ne peut

1 "Un extrait d'une lettre de Racan à Chapelain dans laquelle Racan nous apprend que Malherbe assimilait la prose à la marche, la poésie à la danse." (Valéry, "Propos sur la poésie"). 
analyser un texte poétique comme on analyse, entre autres, un texte narratif ou un discours politique.

Pour une analyse multidimensionnelle du texte poétique, il faudra étudier tous les signes et leurs rapports réciproques. Comme le mentionnait Michael Riffaterre, «le poème est une entité finie et close qui doit être analysée avec une méthode sémiotique » (1983, p. 16) discursive. Pour lui, au-delà de la forme et du sens, il faudra chercher la « signifiance » qui désigne un tout sémantiquement et formellement unifié : « il s'agit de similitudes formelles et positionnelles entre certains mots du texte, similitudes qui sont rationalisées, interprétées en termes de signification » (Riffaterre, 1979, p. 38).

Cet article a pour objectif de proposer une analyse puisée dans la sémantique et la sémiotique poétique. II s'agit d'un poème lyrique de Paul Verlaine (1844-1896) : « Le ciel est, par-dessus le toit » publié dans le recueil Sagesse (1881) :

1. Le ciel est, par-dessus le toit,

2. Si bleu, si calme !

3. Un arbre, par-dessus le toit,

4. Berce sa palme.

5. La cloche, dans le ciel qu'on voit,

6. Doucement tinte.

7. Un oiseau sur l'arbre qu'on voit

8. Chante sa plainte.

9. Mon Dieu, mon Dieu, la vie est là

10. Simple et tranquille.

11. Cette paisible rumeur-là

12. Vient de la ville.

13. Qu'as-tu fait, ô toi que voilà

14. Pleurant sans cesse,

15. Dis, qu'as-tu fait, toi que voilà,

16. De ta jeunesse?

Avant de procéder à une analyse détaillée sur les plans de la forme et du sens, on s'arrêtera sur la structure générale du poème. Du point de vue grammatical, lexical et sémantique, le poème se divise en deux séquences : 
- d'une part les 3 premières strophes où ne figure que le temps présent servant à la description de la nature et de la ville ; et, c'est toujours dans ces strophes que s'accumule un lexique euphorique pour peindre un dehors, un monde extérieur (v. 9, « la vie est là ») ;

- de l'autre, la dernière strophe où paraît le seul temps passé (utilisé deux fois) de manière à exprimer un regret ; en outre quasiment le seul mot dysphorique (v. 14, « pleurant») apparaît dans cette strophe et celui-ci désigne une personne dans un espace coupé du dehors (v. 13, v. 15, « toi que voilà »).

\section{Niveau de la forme}

Nous observons qu'il s'agit d'un poème à forme fixe étant donné que la structure des rimes est régulière quant à la disposition des rimes (1) ainsi qu'à celle des rimes féminines et masculines (2) tandis que la disposition des rimes riches et suffisantes ( 3 ) se distingue légèrement des deux autres. Par ailleurs, le système métrique présente aussi une régularité ferme.

Tableau 1 : Disposition rimique et système métrique

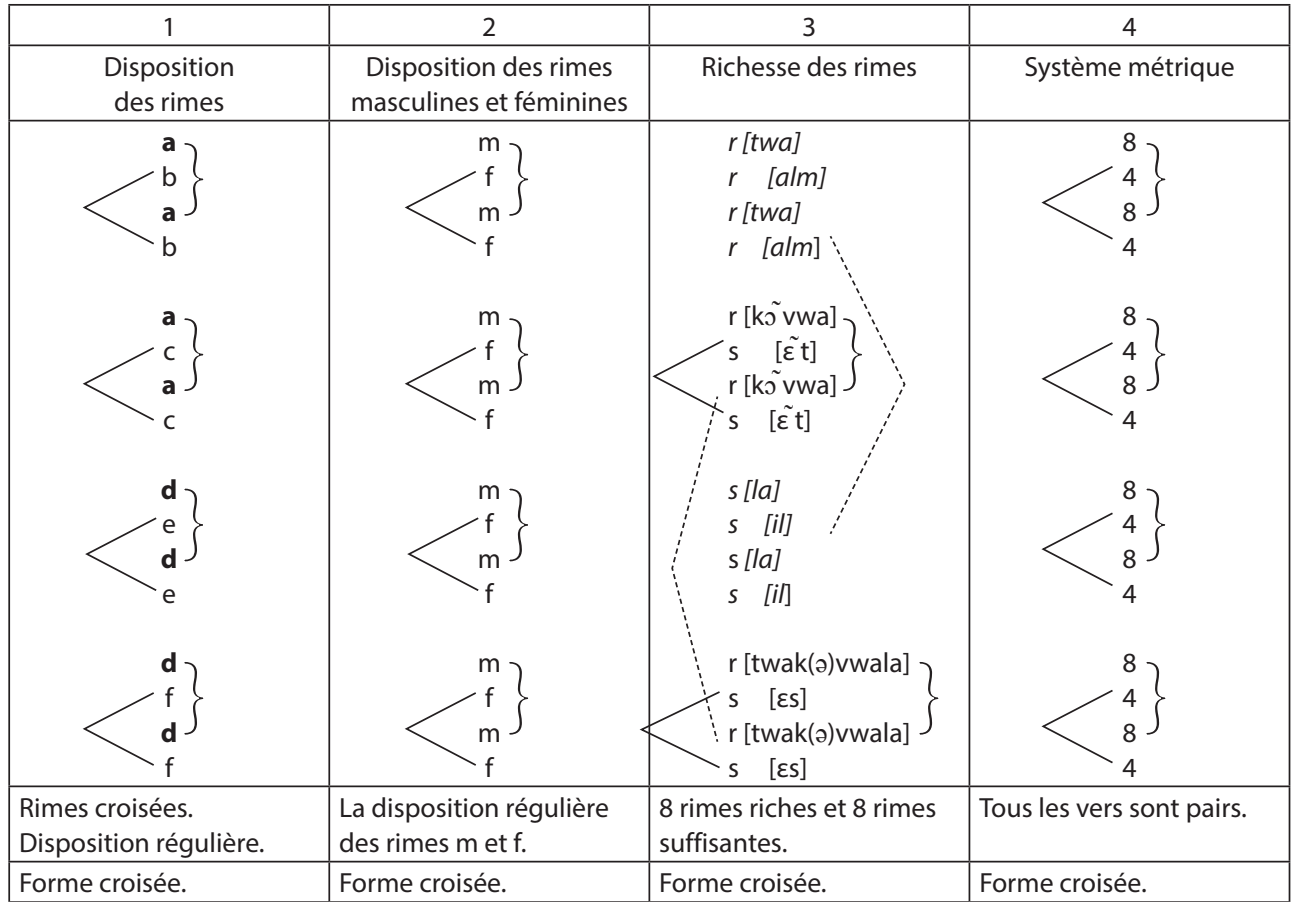


Comme on peut le constater dans le tableau,

1- dans toutes les strophes, il s'agit de rimes croisées. Par surcroit, une des rimes (a) de la $1^{\text {ère }}$ strophe se reprend dans la strophe 2, et une autre (d) de la strophe 3 se reprend dans la strophe 4 ;

2- les rimes masculines et féminines sont également disposées sous forme croisée tout comme dans la première colonne ;

3- la troisième colonne qui montre la richesse des rimes présente une structure différente des deux premières en ce sens que la forme croisée se manifeste deux fois à l'intérieur des strophes ( 2 et 4 ) et 2 fois entre les strophes (1 et 3). Ainsi, les strophes 2 et 4 sont identiques où des rimes riches sont suivies de rimes suffisantes (forme croisée), tandis que dans les $1^{\text {ère }}$ et $3^{3 \text { ème }}$ strophes, la structure des rimes prend une forme plate, à cette différence que la 1 ère strophe contient 4 rimes riches alors que dans la $3^{\text {ème }}$ se succèdent 4 rimes suffisantes. Ainsi, le total des rimes riches est égal à celui des rimes suffisantes : 8 pour chacune ;

4- pour ce qui est de la dernière colonne où figure le système métrique, on observe que tous les vers sont pairs et un long vers de 8 syllabes est suivi d'un autre de 4 syllabes dans l'ensemble du poème. Ce qui correspond une fois encore à la forme croisée.

\section{Les réitérations du signifiant}

Dans cette catégorie, on ne prend en compte que la forme phonique des mots à plus forte raison que les poèmes sont composés avant tout des sons qui s'accordent entre eux. On relève dans ce petit poème plusieurs phonèmes qui se réitèrent nombre de fois.

1) La réitération des phonèmes, qui se présente comme allitération et assonance, assure le rythme dans les poèmes.

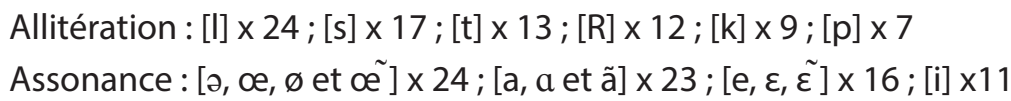

Ces réitérations phoniques d'un nombre élevé permettent d'établir certains liens implicites ou indirects avec le contenu du poème. Ainsi, par l'allitération et l'assonance, 
on peut obtenir des mots et des concepts dont certains figurent dans le poème : « tinte » [tz̃ t], « pleur- » [plœ:R] ; /seul/ [sœl], /pâle/ [pa:l], /las/ [la], /cœur/ [kœ:R], / peur/ [pœ:R], etc.

2) Les homophones et les paronymes font également partie des réitérations du signifiant à cette différence que les derniers sont considérés comme des réitérations partielles :

Les homophones : « toit » - « toi » [twa] / « la » - « là » [la] / « et » - « est » [e] Les paronymes : «calme» - «palme» $[$ kalm - palm] / « vie » - « ville » $[$ vi $\mathrm{vi} /] /$ / voit » - « toit » [vwa - twa].

Il va sans dire que toutes ces réitérations sur le plan de la forme sonore contribuent non seulement à la musicalité et au rythme du poème, mais en même temps à la production d'un certain effet de sens.

\section{Niveau du sens}

En 1960, «Roman Jakobson a identifié la structure de la poésie avec le parallélisme. Selon lui, la fonction poétique se caractérise par la répétition du semblable dans le discours, que ce soit dans l'ordre de la forme ou dans celui du contenu » (Jenny, 2003).

Tout comme au niveau de la forme, les reprises sémantiques aident à créer un certain rythme et à établir des rapports de sens entre les différents mots ou syntagmes. Aussi seront considérées en premier lieu les réitérations de sens :

Réitérations du SIGNIFIÉ : Des parasynonymes : calme - tranquille - paisible

Réitérations du SIGNE : Ce petit poème est composé de très peu de mots (57 seulement, mis à part les articles). Il y figure 27 mots pleins ayant une signification propre contre 13 mots-outils ou mots vides qui n'ont qu'un rôle grammatical ou déictique. Ce qui est à remarquer, c'est que parmi tous ces mots, une grande partie est reprise systématiquement deux fois.

Les mots-outils (22, les répétitions comprises) : Parmi les 13 mots-outils, 9 sont réitérés deux fois : par-dessus (x2), si (x2), sa (x2), mon (x2), là (x2), 
qu' (2x), toi (x2), que (x2), voilà (x2) (d'autres mots-outils figurant sans réitération : dans, sur, et, ta).

Les substantifs (16, les répétitions comprises) : Parmi les 12 adjectifs contenus dans le poème, 4 sont réitérés deux fois : ciel ( $x 2)$, toit ( $x 2)$, arbre (x2), Dieu (x2) (d'autres substantifs figurant sans réitération : palme, cloche, oiseau, plainte, vie, rumeur, ville, jeunesse)

Les verbes (12, les répétitions comprises) : Dans l'ensemble du poème, on relève 9 verbes dont 3 sont réitérés également deux fois : être $(x 2)$, voir $(\mathrm{x} 2)$, faire $(\mathrm{x} 2)$ (d'autres verbes figurant sans réitération : bercer, tinter, chanter, venir, dire, pleurer)

Signalons au passage que contrairement aux substantifs et aux verbes, les adjectifs et les adverbes sont au nombre limité et ne se réitèrent pas. Ainsi, 5 adjectifs qualifient le ciel, la vie et la rumeur : bleu, calme (ciel) ; simple, tranquille (vie); paisible (rumeur de la ville), et 2 adverbes se rapportent au verbe tinter et pleurer : doucement (tinte) / (pleurant) sans cesse.

En outre, plusieurs syntagmes qui se répètent en entier créent une cadence particulière dans l'ensemble de ce poème : par-dessus le toit ( $x 2)$, qu'on voit ( $x 2$ ), mon Dieu (x2), qu'as-tu fait (x2), toi que voilà (x2). Bien qu'il n'y ait pas un refrain qui figure habituellement à la fin des strophes, Verlaine a composé un refrain « partiel » dans la dernière strophe :

(v. 13) Qu'as-tu fait, [ô] toi que voilà

(v. 15) [Dis], qu'as-tu fait, toi que voilà

\section{Les oppositions}

Les oppositions sémantiques et notionnelles établies dans un poème sont souvent très significatives et informatives. Saussure avançait l'idée que « dans la langue, chaque terme a sa valeur par son opposition avec tous les autres termes » (1916, p. 126). En effet, « l'observation d'un élément isolé n'est d'aucune utilité : seul compte le faisceau d'oppositions qui le détermine » (www.universalis.fr). Dans le poème de Verlaine, plusieurs oppositions peuvent être relevées aussi bien au niveau lexical qu'au niveau notionnel : 


\section{Opposition 1 : /extérieur/vs /intérieur/}

Une première opposition qui nous paraît principale se dessine entre /extérieur/ et /intérieur/. On comprend que le /JE/ décrit l'extérieur (« la vie est là ») depuis un espace intérieur (" toi que voilà »). Le /JE/ n'est donc pas loin de la vie extérieure puisqu'il en voit et entend plusieurs éléments : (visuels) ciel, toit, arbre, oiseau ; (auditifs) chant de l'oiseau, tintement de la cloche, rumeur. Pour bien juger de la pertinence de cette opposition, il suffit de montrer les éléments qui font partie de l'espace extérieur et de l'espace intérieur :

Tableau 2 : Opposition spatiale

\begin{tabular}{|l|l|}
\hline Extérieur & Intérieur \\
\hline la /nature/ (« ciel, arbre, palme, oiseau ») & \\
\hline la « ville » (« toit, cloche, rumeur ») & l'état /statique/ (« qu'on voit », « pleurant sans cesse ») \\
\hline la « vie » /dynamique/ (« berce, tinte, rumeur ») & $\begin{array}{l}\text { /la solitude/: monologue (sous la forme d'un dialogue: } \\
\text { toi = moi) }\end{array}$ \\
\hline la « vie» /collective/ (« rumeur ») & $\begin{array}{l}\text { /la vie compliquée/ : /regret/ (« qu'as-tu fait de ta } \\
\text { jeunesse ? ») }\end{array}$ \\
\hline la « vie » « paisible » (« calme, simple, tranquille ») & «pleurer » (/JE/) \\
\hline « chanter » (« oiseau ») & «mon Dieu », « tu »=/JE/ \\
\hline
\end{tabular}

Opposition 2 : /euphorie/vs /dysphorie/

Le poète a renforcé l'opposition spatiale par une opposition thymique entre l'univers /euphorique/ et l'univers /dysphorique/ auquel il appartient :

Tableau 3 : Opposition thymique

\begin{tabular}{|c|l|}
\hline \multicolumn{1}{|c|}{ Euphorie } & \multicolumn{1}{c|}{ Dysphorie } \\
\hline $\begin{array}{l}\text { «Ciel, calme, berce, doucement, simple, tranquille, } \\
\text { paisible » }\end{array}$ & $\begin{array}{l}\text { "pleurant »/JE/ } \\
\text { (« jeunesse » est ici dysphorisée : « qu'as-tu fait de ta } \\
\text { jeunesse?») }\end{array}$ \\
\hline L'univers des autres & \multicolumn{1}{c|}{ L'univers du poète } \\
\hline extérieur & intérieur \\
\hline
\end{tabular}

$\Rightarrow$ Dans la syntaxe du poème, la « rumeur » qui est un mot essentiellement dysphorique est euphorisée par l'adjectif « paisible » alors que «chanter » étant un mot essentiellement euphorique est dysphorisé par «sa plainte ». 
$\Rightarrow$ C'est par voie d'une riche description de l'univers extérieur euphorique d'où il est exclu que le poète met en relief l'univers intérieur dysphorique où il se trouve. En effet, au lieu de décrire son état dysphorique à l'intérieur, il opte, par un procédé inverse, pour décrire l'extérieur euphorique dont il se trouve expulsé.

Opposition 3 : /passé/ vs /présent/

Tableau 4 : Opposition temporelle

\begin{tabular}{|c|c|}
\hline \multicolumn{1}{|c|}{ Passé } & \multicolumn{1}{c|}{ Présent } \\
\hline «jeunesse» (/faute(s)/) & /fin de la jeunesse/ (/regret/) \\
\hline dysphorique & dysphorique \\
\hline
\end{tabular}

Cette opposition temporelle et énonciative entre le /passé/ et le /présent/ est établie par les syntagmes : « qu'as-tu fait de ta jeunesse » (passé) - « toi que voilà » (présent). En outre, la « jeunesse » appartenant au passé (à l'univers extérieur, dans le passé) est en même temps dysphorisée dans la dernière strophe :
Qu'as-tu fait, ô toi que voilà
Pleurant sans cesse,
Dis, qu'as-tu fait, toi que voilà,
De ta jeunesse?

Ce quatrain ayant un ton douloureux exprime, plutôt que le désir d'un retour dans le passé, un regret d'avoir gaspillé la jeunesse, probablement par des fautes importantes. Par conséquent, le temps présent du /JE/ (/la fin de la jeunesse/) est aussi dysphorique que son temps passé (la « jeunesse »).

Opposition 4 : /nature/ vs /culture/

Tableau 5 : Opposition nature/culture

\begin{tabular}{|c|l|}
\hline Nature & \multicolumn{1}{c|}{ Culture } \\
\hline ciel (x2), arbre (x2), palme, oiseau, chanter & $\begin{array}{l}\text { toit (x2), bercer, cloche, tinter, plainte, rumeur, ville, dire, } \\
\text { pleurer }\end{array}$ \\
\hline 5 mots (7 avec les reprises) & \multicolumn{1}{c|}{9 mots (10 avec les reprises) } \\
\hline
\end{tabular}

Cette opposition notionnelle entre /nature/ et /culture/ se cristallise par le lexique du poème. Le nombre des éléments de la culture y est pratiquement le double de celui des éléments naturels, car le poète décrit en particulier, et par un procédé métonymique, 
une ville avec ses bâtiments (« toits »), ses habitants (« rumeur ») et son Église (« cloche »). II décrit en même temps quelques éléments de la nature (partielle) dans la ville, avec un « arbre », sa « palme » et un « oiseau » qui chante. Par ailleurs, le /JE/ qui parle de son état actuel recourt aux mots ayant un contenu culturel. En effet, tandis que « dire» et « pleurer » sont des actes propres aux humains, « la plainte » étant un acte culturel est ici attribué à l'oiseau.

\section{Opposition 5 : /les autres/vs /JE/}

Une dernière opposition s'établit en partant du mot-clé « la rumeur » signifiant un «bruit confus produit par la présence d'un certain nombre de personnes qui parlent, crient ou s'activent plus ou moins loin » (cnrtl). Dans le sémantisme de ce mot, on distingue les sèmes /bruit/, /nombreuses personnes/, /parler, crier, s'activer/, /plus ou moins loin/, et qui s'opposent ainsi au /JE/ demeurant dans sa solitude :

Tableau 6 : Opposition /les autres/ / /JE/

\begin{tabular}{|c|c|c|}
\hline & /les autres/ & /JE/ \\
\hline & Les gens dans un espace ouvert d'où vient une « rumeur ». & $\begin{array}{l}\text { /Seul/ (dans un espace probablement } \\
\text { fermé) }\end{array}$ \\
\hline & Ils parlent, crient ou s'activent plus ou moins loin (du /JE/) & $\begin{array}{l}\text { Ne fait que «pleurer » et /culpabiliser/ } \\
\text { dans un état passif. }\end{array}$ \\
\hline 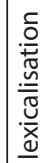 & «rumeur» & $\begin{array}{l}\text { « Mon » (Dieu) }(x 2), \text { «tu » }(x 2)=j e ; \text { « toi » } \\
=\text { je } ; \text { «ta »= ma ; « pleurer » }\end{array}$ \\
\hline
\end{tabular}

Les 5 couples opposés que nous avons relevées se présentent donc comme suit :

Tableau 7 : Ensemble des oppositions

\begin{tabular}{|l|l|c|}
\hline Oppositions & \multicolumn{2}{|c|}{} \\
\hline 1 & /Extérieur/ & /Intérieur/ \\
\hline 2 & /Euphorie/ & /Dysphorie/ \\
\hline 3 & /Passé/ (dysphorisé) & /Présent/ \\
\hline 4 & /Culture/ + /Nature/ & /Culture/ («pleurer ») \\
\hline 5 & /Les autres/ (actifs) & /JE/ (passif) \\
\hline
\end{tabular}

\section{Les rapports référentiels entre les mots}

Un des procédés sémantiques et sémiotiques porte sur le réseau de relations entre différents signes d'un texte. Comme nous le verrons ci-dessous, un même signe peut 
avoir une correspondance avec plus d'un signe. Dans ce poème, on constate que nombre de mots et de syntagmes se font écho, ce qui assure une pertinence sémantique via des champs associatifs :

1) «Berce » (v. 4) renvoie à « chante » (v. 8) du fait que les deux actes se relient par un sème commun :/rythme/.
(v. 4) Berce sa palme.
[sujet : arbre] (+ animé)
(v. 8) Chante sa plainte.
[sujet : oiseau] (+ animé)

2) «Tinte » (v. 6), « chante » (v. 8) et « rumeur » (v.11) se rapprochent par leur sème commun /son, bruit/ ou /sens auditif/ :
(v. 6) Doucement tinte.
[sujet : cloche] (-animé ; +culture)
(v. 8) Chante sa plainte.
[sujet : oiseau] (+animé ; -culture)
(v. 11) Cette paisible rumeur-là
[source : ville] ( \pm animé ; \pm culture)

3) La « plainte » (v. 8) de l'oiseau réfère au dernier quatrain, par un rapport d'opposition actionnelle, à « pleurant » (v. 14) étant donné que les deux mots se retrouvent autour de la valeur/dysphorique/ :
(v. 8) Chante sa plainte.
[sujet : oiseau] (+animé ; -humain)
(v. 14) Pleurant sans cesse,
[sujet : tu $=/ \mathrm{JE} /$ ] (+animé ; +humain)

4) La « cloche » (v. 5) de /l'église/ se rapporte, par métonymie, à « Mon Dieu, mon Dieu » (v. 9) par le sème /religion/:

(v. 5) La cloche, dans le ciel qu'on voit, [partie-tout:cloche-église] (-animé;+culture)

(v. 9) Mon Dieu, mon Dieu, la vie est là [sujet parlant :/JE/] (+animé ; +culture)

5) Par ailleurs, plusieurs mots de catégories différentes se font également écho par leur sémantisme ou leur valeur thymique : ainsi « calme » (v. 2), « berce » (v. 4), « doucement » (v. 6), « simple », « tranquille » (v. 10) et « paisible» (v. 11) se relient-ils par le concept de /douceur/ ou par une /évocation agréable/, donc par la valeur euphorique :

$\begin{array}{lll}\text { (v. 2) Si bleu, si calme! } & \text { [ciel] } & \text { (+nature) } \\ \text { (v. 4) Berce sa palme. } & \text { [arbre] } & \text { (+nature) } \\ \text { (v. 6) Doucement tinte. } & \text { [cloche] } & \text { (+culture) } \\ \text { (v. 10) Simple et tranquille. } & \text { [vie] } & \text { (+nature ;+culture) } \\ \text { (v. 11) Cette paisible rumeur-là } & \text { [ville] } & \text { (+culture) }\end{array}$


6) Enfin, par un rapport d'opposition spatiale, le syntagme « la vie est là » (v. 9) se rattache à « toi que voilà » (v. 13 et 15).

(v. 9) Mon Dieu, mon Dieu, la vie est là $\quad[$ vie = là-bas $] \quad$ (+nature ; +culture)

(v. 13) Qu'as-tu fait, ô toi que voilà $\quad[« t u »=/ J E /=$ ici $] \quad$ (+culture)

(v. 15) Dis, qu'as-tu fait, toi que voilà, $\quad[« t u »=/ J E /=$ ici $] \quad$ (+culture)

\section{L'état et la place du /JE/ :}

Dans les trois premières strophes, le /JE/ du poème décrit l'extérieur tandis que seule la dernière strophe est consacrée au monde intérieur où il semble se trouver coincé.

Figure 1 : Présentation des espaces par rapport au /JE/

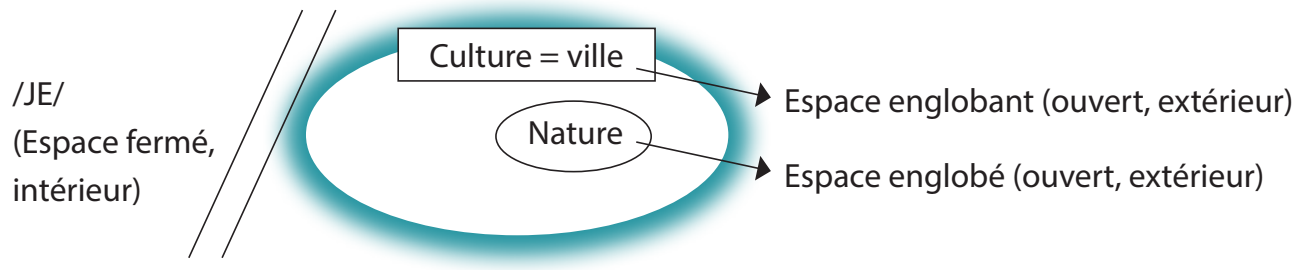

Bien que le /JE/ se trouve proche de l'espace extérieur, il est coupé de ces deux espaces ouverts puisqu'il regarde et entend depuis un endroit intérieur où il semble enfermé. Il lui est donc impossible de faire partie de l'espace ouvert qu'il décrit comme un monde euphorique.

Par ailleurs, la présence du /JE/ poétique (ou la persona lyrique) ne se lexicalise que par le « on » qui le renferme, par l'interjection « mon Dieu » et par « toi » en s'adressant à lui-même.

\section{Les figures de style}

Dans l'ensemble du poème, on peut relever deux métaphores et un oxymore. Dans la première strophe, il s'agit d'une métaphore filée dans le syntagme « un arbre [...] berce sa palme » : au sens propre, le verbe « bercer » s'emploie par un sujet-agent humain et un complément-bénéficiaire également humain, plus précisément, un bébé ou un enfant. Tandis que dans les vers 3 et 4 , le sujet est un non-humain (« arbre ») ainsi que le complément (« palme») : 
arbre ...BERCE sa palme (« arbre » : -humain / « berce » : + humain / «palme»: -humain)

Dans la strophe 2, une autre métaphore a été créée également par deux traits lexicaux qui s'opposent. Ainsi, l'acte de se plaindre, propre aux humains, est attribué ici à un oiseau :

un oiseau ... chante sa plainte (« oiseau » :-humain / «plainte »: +humain)

Dans la strophe 3, on observe un oxymore, figure par laquelle le poète a allié « paisible » et « rumeur », deux mots qui s'excluent ordinairement. Et c'est par deux valeurs thymiques opposées (euphorie vs dysphorie) que l'on peut expliquer cet oxymore :

cette paisible rumeur-là (« paisible » : euphorique + « rumeur » dysphorique)

\section{Le contenu du poème}

Pour terminer, à la lumière de l'analyse réalisée, prenons l'ensemble du poème par ordre strophique pour voir la distribution et la progression des éléments analysés dans chaque strophe :

1ere strophe : Le poème débute par la description d'une nature fragmentaire (dans la ville), faite par le sens visuel : le toit, le ciel (bleu ; calme). Il faut rappeler que c'est une vue restreinte et qu'il ne s'agit nullement de la description d'un paysage en entier. Par surcroit, «l'arbre» qui « berce sa palme » présuppose la présence d'un vent léger.

2eme strophe : La première description est suivie d'une autre, qui porte cette fois-ci sur le sens auditif : le /JE/ entend le tintement de la cloche et le chant d'un oiseau : « La cloche (...) doucement tinte »; « un oiseau (...) chante sa plainte ». Notons de passage que les mots ciel, cloche et oiseau dans cette strophe créent un univers euphorique, à l'exception de la « plainte », mot à valeur dysphorique.

3eme strophe : C'est l'interjection «Mon Dieu » qui désigne la première apparition lexicale du /JE/. Cette interjection réitérée est difficilement interprétable : montre-t-elle tout simplement un émerveillement devant la simplicité et le calme de l'univers extérieur ainsi que de la vie des autres, 
ou traduit-elle le sentiment de regret exprimé dans la dernière strophe? La seconde hypothèse peut être étayée par l'opposition qui relie les deux strophes : «La vie est là » (strophe 3) / «Toi que voilà » (strophe 4). Ainsi, l'interjection dans la strophe 3 semble exprimer simultanément l'admiration devant la vie extérieure et le regret d'en avoir été exclu par sa propre faute, s'il en est.

4eme strophe : A la suite de la première apparition du /JE/, c'est un « tu » qui se manifeste. Ainsi, le monologue de la strophe précédente se transforme en une forme de dialogue dont les participants semblent être la même personne. Le /JE/ se pose de la sorte une question rhétorique : «Qu'as-tu fait (...) de ta jeunesse ? ». Cette même question présuppose qu'il n'est plus jeune, et qu'il regrette et culpabilise d'avoir gâché sa jeunesse («Qu'as-tu fait, ô toi que voilà »). Comme il a été indiqué plus haut, le /JE/ se présente dans cette dernière strophe dans l'espace intérieur (« toi que voilà » par opposition à « la vie est là »).

Tableau 8 : Tableau récapitulatif de l'ensemble du poème

\begin{tabular}{|c|l|l|l|l|l|l|}
\hline strophe & \multicolumn{1}{|c|}{ nature } & \multicolumn{1}{c|}{ culture } & \multicolumn{1}{c|}{ visuel } & \multicolumn{1}{c|}{ auditif } & \multicolumn{1}{|c|}{ je / autres } & euphorie / dysphorie \\
\hline $\mathbf{1}$ & $\begin{array}{l}\text { ciel, arbre, } \\
\text { palme }\end{array}$ & toit & $\begin{array}{l}\text { ciel, arbre, } \\
\text { palme }\end{array}$ & & autres & euphorie (extérieur) \\
\hline $\mathbf{2}$ & ciel, oiseau & cloche & ciel, arbre & $\begin{array}{l}\text { cloche, } \\
\text { tinte, chante }\end{array}$ & autres & euphorie (extérieur) \\
\hline $\mathbf{3}$ & vie & ville, Dieu & & rumeur & $\begin{array}{l}\text { /JE/ ('mon Dieu') + } \\
\text { /autres/ ('rumeur') }\end{array}$ & euphorie (extérieur) \\
\hline $\mathbf{4}$ & & & & pleurer & " tu », «toi »=/JE/ & dysphorie (intérieur) \\
\hline
\end{tabular}

\section{Conclusion}

Sur le plan formel, nous avons montré que toutes les dispositions rimiques ainsi que le système métrique se concordaient presque parfaitement. Ce parallélisme établi entre les différents niveaux de forme témoigne de la solidité de la structure textuelle. En outre, l'harmonie des sons produit un rythme régulier et agréable (bien que triste) de manière à refléter le contenu du poème : un univers euphorique relié à l'état affectif dysphorique du je poétique.

Sur le plan du contenu, comme il a été mentionné dans l'introduction et dans le tableau 8 , ce poème de quatre strophes se divise en deux séquences : les 3 premières strophes décrivent le monde extérieur euphorisé par tous ses éléments constitutifs, 
tandis que la dernière strophe est réservée au monde intérieur où se trouve le /JE/. Cet univers intérieur est complètement dysphorique comme le montre un syntagme répété deux fois («Qu'as-tu fait ? ») exprimant le regret étayé par le verbe « pleurer ». Le poète a ainsi consacré 3 strophes au monde extérieur qu'il a euphorisé pour souligner, par voie de contraste, son petit monde dysphorique en une seule strophe. De là, on peut supposer que par rapport à un espace /ouvert/ et /étendu/ (ciel, arbre, ville, etc.), il demeure dans un espace /fermé/ ou /réduit/. Bien que le /JE/ se trouve dans la ville qu'il décrit (puisqu'il en voit et entend plusieurs éléments), il semble être dans l'impossibilité de participer à la vie commune. En plus, quand il décrit la ville, on comprend qu'il a une vision très restreinte, car il ne voit qu'une partie de ciel, de toit et d'arbre.

L'originalité d'un tel procédé provient d'un choix artistique : dans les trois premières strophes, le poète s'est servi abondamment de la description d'un monde euphorique afin de mettre l'accent sur son état dysphorique dans la dernière strophe. A côté de la musicalité remarquable dans l'ensemble de l'œuvre de Verlaine, c'est notamment par cette structure reposée sur les oppositions sur les plans sémantique et notionnel que l'on peut caractériser ce poème.

Évaluation : Évaluation anonyme par des pairs extérieurs.

Conflit d'intérêts : L'auteure n'a aucun conflit d'intérêts à déclarer.

Subvention : L'auteure n'a reçu aucun soutien financier pour ce travail.

Peer-review: Externally peer-reviewed.

Conflict of Interest: The author has no conflict of interest to declare.

Grant Support: The author declared that this study has received no financial support.

\section{Bibliographie}

Jakobson, R. (1963). Essais de linguistique générale. (trad. par Nicolas Ruwet). Paris : Minuit.

Jakobson, R. (1973). Questions poétiques. Paris : Seuil.

Jenny, L. (2003). La poésie. Méthodes et problèmes.

URL : https://www.unige.ch/lettres/framo/enseignements/methodes/elyrique/elintegr.html (Consulté le 03.01.2020)

Riffaterre, M. (1979). La production du texte. Paris : Seuil.

Riffaterre, M. (1983). Sémiotique de la poésie. (Traduit de l'anglais par Jean-Jacques Thomas). Paris : Seuil.

Saussure, F. de (1916). Cours de linguistique générale. Paris : Payot.

Valéry, P. (1928). Propos sur la poésie. Paris : Gallimard (1957).

Verlaine, P. (1881). Sagesse. Paris : Livre de poche (2003).

www.universalis.fr (Consulté le 03.02.2020) 
